\title{
Das Setzen und Verwirklichen von Zielen
}

\author{
Gabriele Oettingen \\ Universität Hamburg \\ Peter M. Gollwitzer \\ Universität Konstanz
}

\begin{abstract}
Zusammenfassung: Die historischen Wurzeln des Zielkonzepts werden im Behaviorismus, im Mentalismus sowie in der deutschen Willenspsycholgie gesucht. Es wird darauf hingewiesen, daß die Psychologie des Zielstrebens in den letzten 20 Jahren eine Wiederbelebung erfahren hat. Die moderne Zielpsychologie beschäftigt sich mit Phänomenen der Zieisetzung und der Zielverwirklichung. In Hinblick auf die Zielsetzung werden sowohl inhaltstheoretische Ansätze als auch selbstregulatorische Ansätze diskutiert. Die inhaltstheoretischen Ansätze versuchen, erfolgreiche Zielsetzung aufgrund von prägnanten Zielmerkmalen vorherzusagen (z. B. fremdgesetzte versus selbstgesetzte Ziele, konkrete versus abstrakte Ziele, Ideal- versus Sollziele, Leistungs- versus Lernziele). Die selbstregulatorischen Ansätze dagegen untersuchen Prozesse, die eine erfolgreiche Zielsetzung begünstigen ( $z$. B. mentale Kontrastierung von Zukunft und Realität, Stärkung von Selbstwirksamkeitserwartungen). Schließlich kommt die Verwirklichung von Zielen zur Sprache. Auch hier werden inhaltliche oder strukturelle Zielmerkmale (z. B. materielle versus ideelle Ziele, proximale versus distale Ziele, Annäherungs- versus Vermeidungsziele) einerseits und selbstregulatorische Prozesse (z. B. planende Bewußtseinslage, Vorsatzbildung, Handlungskontrollstrategien, Anstrengungssteigerung,

Diskrepanzreduktion) andererseits identifiziert. Abschiießend werden zielpsychologische Phänomene diskutiert, die bislang in der Psychologie des Zielstrebens weitgehend unberücksichtigt geblieben sind ( $z$. B. Zielkonflikte, das Aufgeben von unpassenden Zielen).
\end{abstract}

\section{Schlüsselwörter: Zielsetzung, Zielverwirklichung, Handlung, Selbstregulation, Zielmerkmaie}

\section{Goal Setting and Goal Implementation}

\begin{abstract}
Summary: The historical roots of the goal concept are found in behaviorism, mentalism, and German will psychology. It is suggested that the psychology of goal pursuit has recently experienced a renaissance. Modern goal psychology focuses on phenomena of goal setting and goal attainment. With respect to goal setting, content approaches as well as self-regulatory approaches are differentiated. Goal content approaches try to predict successful goal setting on the basis of distinct features (e. g., assigned versus self-set goals, specific versus abstract goals, promotion versus prevention goals, performance versus learning goals). Self-regulatory approaches, to the contrary, analyze processes that facilitate successful goal setting (e. g., mental contrasting of the future with impeding reality, promoting selfefficacy expectations). Further, issues of goal implementation are investigated. Again, relevant content and structural features of goals (e. g., materialistic versus idealistic goals, proximal versus distal goals, approach versus avoidance goals) and self-regulatory processes (e. g., implemental mindsets, implementation intentions, action control strategies, energization, discrepancy reduction) are identified. Finally, goai relevant phenomena which have been widely ignored in modern goal psychology (e. g., goal conflict, goal disengagement) are addressed.
\end{abstract}

Keywords: Goal setting, goal implementation, action, self-regulatory processes, goal contents

Im letzten Jahrzehnt hat die Frage nach dem Entstehen von Zielen und mehr noch nach der Verwirklichung von Zielen großes Interesse erfahren. Dies ist durch die Veröffentíchung verschiedener Sammelbände (z. B. Frese \& Sabini, 1985; Gollwitzer \& Bargh, 1996; Martin \& Tesser, 1996; Pervin, 1989) und Überblicksarbeiten (z. B. Gollwitzer \& Moskowitz, 1996; Karniol \& Ross, 1996; Karoly, 1993; Austin \& Vancouver, 1996) dokumentiert. Über die Ursachen läßt sich nur spekulieren. Eine davon dürften die neuen theoretischen Entwicklungen in der Motivations-psychologie sein (Geen, 1995; Gollwitzer, 1990; Heckhausen, 1991; Kuhl, 1984). Dort war man bislang davon ausgegangen, daß die Bereitschaft, ein bestimmtes Verhalten auszuführen, ausreichend durch die Schlüsseldeterminanten Erwartung und Anreiz bestimmt ist. Auf der Grundlage von Erwartungen und Anreizen motiviert zu sein, reicht jedoch nicht aus, um eine erfolgreiche Handlungsausführung zu gewährleisten. Diese kann selbst bei hoher Motivation dadurch bedroht sein, daß bspw. der Gebrauch geeigneter Gelegenheiten und Mittel nicht geplant, Ablenkungen nicht ignoriert, unterbrochenes Zielstreben nicht wiederaufgenommen, auf Schwierigkeitssteigerungen nicht mit erhöhter Anstrengung reagiert und Mißerfolge nicht bewältigt werden (Mischel, Cantor \& Feldman, 1996). In den genannten Fällen muß willentliche Kontrolie über das Handeln ausgeübt werden. Dies kann dadurch geschehen, daß man sich geeignete Ziele setzt und deren Realisierung effektiv vorantreibt.

Im vorliegenden Beitrag werden verschiedene Determinanten und Prozesse der Zielsetzung einerseits und der Zielrealisierung andererseits diskutiert. Wir werden zunächst fragen, welche personbezogenen und situativen Variablen das Setzen von Zielen begünstigen und auf welchen psychologischen Prozessen die Zielsetzung beruht. Danach erörtern wir, welche Qualitäten gesetzter Ziele die Zielrealisierung erleichtern und welche selbstregulatorischen Strategien besonders nützlich sind. 


\section{Die Geschichte des Zielkonzepts}

Behavioristen erkennen zielgerichtetes Verhalten an seinen Merkmalen. Zielgerichtetes Verhaiten ist zunächst persistent. Eine hungrige Ratte bspw. sucht den Käfig so lange ab, bis sie die versteckte Nahrung entdeckt hat (Tolman, 1925). Zielgerichtetes Verhalten ist obendrein angemessen in dem Sinne, daß bei der Blockierung eines bestimmten Wegs zum Ziel sofort ein alternativer Weg gewählt wird, oder falls sich das Ziel bewegt (z. B. die Maus vor der sie verfolgenden Katze), diesem entsprechend gefolgt wird. Schließlich zeichnen sich zielgerichtete Organismen dadurch aus, daß sie angesichts relevanter Kontextstimuli nach dem Zielobjekt zu suchen beginnen.

Die Aussage eines behavioristischen Forschers, daß ein Stück Nahrung für einen hungrigen Organismus (z. B. eine Ratte) ein Ziel ist, bedeutet nicht mehr, als daß dieses Stück Nahrung einen Anreiz darstellt, und der Forscher sich entschieden hat, das Verhalten der Ratte in Relation zu diesem Nahrungsstimulus zu beschreiben und nicht in Relation zu einem anderen Objekt oder Ereignis. Skinner (1953) bezeichnete deshalb Zielgerichtetheit als eine bequeme Kurzform der Beschreibung von Verhalten, das aufgrund irgendeines operanten Konditionierungsprozesses entstanden ist. Festzuhalten ist, daß in der behavioristischen Tradition der Bezugspunkt zielgerichteten Verhaltens nicht das vom Organismus selbst gesetzte Ziel ist (Bindra, 1959).

Im Gegensatz dazu ist der Bezugspunkt der heutigen Zieltheorien das internale subjektive Ziel. Zielgerichtetes Handeln bezieht sich auf Ziele, gegenüber denen die Person sich verpflichtet fühlt (z. B. das Ziel, das Rauchen aufzugeben dient als Bezugspunkt für die Analyse der Anstrengungen, die unternommen werden, um das Ziel nicht mehr zu rauchen, zu erreichen). Forschungsfragen fokussieren auf die Art und Weise, wie und welche Ziele gesetzt werden, und wie die Zielsetzung das Verhalten beeinflußt. Die behavioristische Unterscheidung zwischen Bedürfnissen, Anreizen und zielgerichtetem Verhalten findet sich jedoch auch in heutigen Zieltheorien wieder. Bedürfnisse (z. B. nach Anerkennung) werden dahingehend verstanden, daß sie eine Klasse von Anreizen benennen (z. B. beliebt oder erfolgreich zu sein), wobei Verhaltensziele im Dienste der Erreichung dieser Anreize gesehen werden. Geen (1995) bspw. definiert Anreiz als ein erwünschtes Handlungsergebnis, auf das sich verschiedene Handiungsziele beziehen können. Die Anreize (z. B. beliebt oder erfolgreich sein) werden als Produkte der Bedürfnisse einer Person (d. h. dem Bedürfnis nach Anerkennung) und der wahrgenommenen situativen Möglichkeiten ( $z$. B. dem Bekanntenkreis bzw. der wissenschaftlichen Öffentlichkeit) betrachtet. Die übergeordneten Zielsetzungen, beliebt bzw. erfolgreich zu sein, werden schließlich durch eine Vielzahl von Verhaltenszielen bedient ( $z$. B. die Zielsetzung, das Wochenende mit seinen Bekannten zu verbringen bzw. einen brillanten Artikel zu schreiben).

Die heutige Perspektive, zielgerichtetes Handeln in Relation zu subjektiven Zielen zu analysieren, hat seine eigenen historischen Vorläufer: Die Mentalisten William James and William McDougall in den Vereinigten Staaten einerseits und die Willenspsychologen Narziß Ach und Kurt Lewin in Deutschland andererseits. In seinen Principles of Psychology (1890/1950) führte James aus, daß Verhalten durch gute Absichten gesteuert werden kann, auch wenn diese Steuerung zeitweise nicht gelingen mag. Sind jedoch bestimmte Voraussetzungen (preliminaries) erfüllt, würde das in guten Absichten spezifizierte Verhalten in die Tat umgesetzt werden. McDougall (1908/1931) postulierte, daß Zielsetzungen das Verhalten einer Person durch kognitive Prozesse, die sich auf die Diskrepanz zwischen der vorliegenden Situation und dem gesetzten Ziel beziehen, steuern. Fortschritte in der Zielrealisierung sowie die Zielrealisierung selbst würden positiv erlebt, Rückschläge sowie erlittener Mißerfolg dagegen negativ.

Die wissenschaftliche Analyse des Zielstrebens war in Deutschland geprägt von der Kontroverse zwischen Ach und Lewin. Ach (1935) ging davon aus, daß die mentale Verknüpfung antizipierter zukünftiger Handilungsgelegenheiten mit einem intendierten Verhalten eine Determination entstehen läßt, welche die Person dazu drängt, das Verhalten auszuführen, wenn die kritische Gelegenheit tatsächlich auftritt. Die Stärke der Determination würde davon abhängen, wie konkret die antizipierte Situation spezifiziert ist und wie stark das Wolien der Person ist. Ein bewußter Willensakt würde für die Initiierung des intendierten Verhaltens angesichts der kritischen Situation (Gelegenheit) nicht mehr notwendig sein. Lewin (1926) kritisierte diese Koppelungstheorie der Zielsetzung und Zielrealisierung und offerierte als Alternative eine Bedürfnistheorie. Danach weisen Ziele Objekten und Ereignissen einen Aufforderungscharakter zu. Ähnlich wie bei echten Bedürfnissen (z. B. Hunger), die durch eine Vielzahl von Handlungen befriedigt werden können (z. B. Obst, Brot oder Gemüse essen), würden auch Quasi-Bedürfnisse (Zielsetzungen) durch eine Vielzahl von Handlungen befriedigt werden können (z. B. Freunde zu einem Fest einladen, Geburtstagsgeschenke machen). Die mit einem Quasi-Bedürfnis assoziierte Spannung würde die Stärke des Zielstrebens bestimmen. Die Höhe der Spannung ist abhängig von der Stärke relevanter übergeordneter Bedürfnisse (biologische Bedürfnisse, relevante Lebensziele) und wie eng letztere mit dem vorliegendem Quasi-Bedürfnis in Beziehung stehen. Lewins Metapher des Spannungszustands von Quasi-Bedürfnissen trägt der Tatsache Rechnung, daß es meist mehrere Wege zum gesetzten Ziel gibt, die alternativ oder ersatzweise gewählt werden können.

Viele der von James, McDougall, Ach, Lewin und den Behavioristen vorgetragenen Überlegungen zum Thema Zielstreben sind in die heutigen Zieltheorien eingegangen, wobei allerdings gilt, daß Fragen der Realisierung gesetzter Ziele größere Aufmerksamkeit erhalten haben als Fragen der Zielsetzung. Karoly (1993; "the study of goals as dependent variables remains infrequent") und Carver und Scheier (1999; "the question of where goals come from and how they are synthesized is one that has not been well explored ...") beklagen explizit diese Forschungslücke. Wir werden deshalb mit der Analyse der vernachlässigten Frage beginnen, welche Bedingungen und Prozesse Zielsetzungen zugrundeliegen.

\section{Zielsetzung}

\section{Determinanten der Zielsetzung}




\section{Aufgetragene Zielsetzungen}

Ziele werden einem häufig von anderen aufgetragen (z. B. von Eltern, Lehrern und Arbeitgebern). Ob aufgetragene Ziele als persönlich verbindliche Zielsetzungen übernommen werden, hängt davon ab, wer wem Ziele aufträgt, und wie die entsprechenden Zielstiftungsversuche gestaltet sind. Die relevanten Variablen beziehen sich dabei auf Merkmaie des Einfiußnehmers, des Rezipienten sowie der vorgetragenen Argumente (McGuire, 1969). Seitens des Einflußnehmers sind die Variablen Legitimation und Vertrauenswürdigkeit für die Transformation des aufgetragenen in ein persöniich verbindliches Ziel bedeutsam (Locke \& Latham. 1990). Seitens des Rezipienten ist wichtig, ob das angetragene Ziel als wünschenswert und machbar erscheint, und inwieweit es gelingt, das angetragene Ziel für die eigene Person umzuformulieren und in bereits bestehende Zielsetzungen zu integrieren (Cantor \& Fleeson, 1994). Seitens der vorgetragenen Argumente, schließlich, kommt es u. a. darauf an, daß die Diskrepanz zwischen dem aufgetragenen Ziel und der zugehörigen persönlichen Zielsetzung nicht allzu groß ausfällt, und daß Furchtappelle nur gemeinsam mit Informationen über den Weg zum Ziel eingesetzt werden (z. B. wenn die dramatischen Konsequenzen von gesundheitsschädigendem Verhalten geschildert werden, sollten gleichzeitig Informationen über die Vermeidung gesundheitsschädigenden Verhaltens gegeben werden). Zielstifter müssen auch die Motivation und die Informationsverarbeitungskompetenz des Rezipienten beachten, da diese beiden Variablen sich als Moderatoren der Effekte von Einflußnehmer-, Rezipient- und Argumentmerkmalen auf die persönliche Übernahme von aufgetragenen Zielen auswirken dürften (Petty \& Cacioppo, 1986; Chaiken, 1987).

\section{Selbstgesetzte Ziele}

Ziele müssen nicht aufgetragen werden, sie können auch selbst gesetzt werden. Selbstgesetzte Ziele unterliegen jedoch oft dem Einfluß anderer Personen. So können Ziele gemeinsam gesetzt ( $z$. B. bei gemeinsamer Entscheidungsfindung oder Mitbestimmung; Wilpert, 1994) oder von geachteten Personen übernommen werden (z. B. Wenn vom Versuchsieiter Standards für die eigene Belohnung übernommen werden; Mischel \& Liebert, 1966). Nach Cantor und Fleeson (1991) können auch kontextuelie Stimuli, wie bspw. durch den Kontext vermittelte normative Erwartungen, die Zielsetzung beeinflussen.

Die für die Zielauswahl bedeutsamsten personseitigen Variablen sind Wünschbarkeit und Machbarkeit. Wünschenswerte und machbare Ziele werden am häufigsten gewählt (Ajzen, 1985; Heckhausen, 1991; Gollwitzer, 1990; Locke \& Latham, 1990). Wünschbarkeit entspricht dem eingeschätzten Anreiz der wahrscheinlichen kurz- und langfristigen Konsequenzen der Zielerreichung. Diese Konsequenzen können sich auf antizipierte Selbstbewertungen, auf Bewertungen seitens geschätzter anderer Personen, auf die Annäherung an ein übergeordnetes Ziel, oder auf fremdthematische Belohnungen beziehen (Heckhausen. 1977). Weiterhin dürfte die antizipierte Freude, die das Zielstreben selbst bereiten kann, die Beurteilung der Wünschbarkeit positiv beeinfiussen. Die Machbarkeit entspricht der Kompetenz, die man sich in Hinblick auf die erfolgreiche Ausführung zieigerichteten Verhaltens zuschreibt (d.h. den Selbstwirksamkeitserwartungen; Bandura, 1997), der Überzeugung, daß das zielgerichtete Verhalten auch tatsächlich zur Zielerreichung führt (den Ergebniserwartungen; Bandura, 1997), der generellen Einschätzung, das Ziel zu erreichen (den generalisierten Erwartungen; Oettingen, 1996, 1997a) sowie der Einschätzung, daß die persönliche Zukunft positiv ausfallen wird (dem generellen Optimismus; Scheier \& Carver, 1985). Bei der Feststellung der Wünschbarkeit und Machbarkeit eines potentielien Ziels wird dieses nicht isoliert, sondern in Relation zu anderen potentiellen Zielen gesehen. Ein Ziel mit attraktiven Konsequenzen mag nach dem Bedenken übergeordneter Ziele weit weniger wünschenswert oder in Verbindung mit anderen parallelen Zieien piötzlich machbarer erscheinen (Cantor \& Fleeson, 1994; Gollwitzer, 1990).

Die eingeschätzte Wünschbarkeit und Machbarkeit bestimmen, mit welchem Schwierigkeitsgrad sich Personen Ziele setzen. Festinger (1942) argumentierte in seiner Theorie der resultierenden Valenz, daß Personen denjenigen Schwierigkeitsgrad auswählen, bei dem die erwartete resultierende Valenz am höchsten ist, wobei der Schwierigkeitsgrad das Produkt der Erfolgs/Mißerfolgswahrscheinlichkeit multipliziert mit dem Erfolgs/Mißerfolgsanreiz darstellt. Atkinsons (1957) Risikowahl-Modell modifizierte und erweiterte Festingers Theorie, so daß sich unterschiedliche Vorhersagen für Personen mit Hoffnung auf Erfolg und Personen mit Angst vor Mißerfolg treffen lassen. Während letztere leichte und schwere Aufgaben wählen, entscheiden sich erstere für Ziele mittleren Schwierigkeitsgrades.

Ziele können sich auch in anderen Strukturmerkmalen als dem Schwierigkeitsgrad unterscheiden (z. B. abstrakt vs. konkret) und sie können in ihrem Inhalt variieren (z. B. materiell vs. zwischenmenschlich). Nach der Theorie der Handlungsidentifikation (Valiacher \& Wegner, 1987) denken Personen über ihre Handlungen primär in abstrakten Konzepten nach (z. B. die Wohnung putzen). Sie nutzen erst dann konkrete Konzepte (z. B. den Teppichboden staubsaugen), wenn Schwierigkeiten auftreten. Demnach dürften konkrete Ziele verstärkt dann gesetzt werden, wenn es um Handlungsbereiche geht, die Realisierungsprobleme aufwerfen. Manche Personen denken allerdings generell über ihre Handlungen eher konkret als abstrakt nach (Vallacher \& Wegner. 1989). Diese Vorliebe, Handlungen eher konkret als abstrakt zu begreifen, sollte zur bevorzugten Setzung von konkreten Zielen führen.

Ziele können positiv oder negativ formuliert werden. Sie können sich auf das Erreichen und den Erhalt positiver Zustände oder auf das Vermeiden und die Linderung negativer Zustände beziehen. Nach Higgins (1997). unterscheiden sich Personen darin, inwieweit sie ihr Selbst als ein Ideal begreifen, das sie erreichen wollen, oder als etwas, das sie erreichen "sollen" oder "müssen". Während das Ideal-Selbst auf das Erreichen von Fortschritten abhebt, ist das Soll-Selbst auf die Vermeidung von Rückschritten ausgerichtet. Das Ideal-Selbst solite demnach dazu führen, sich positiv formulierte Ziele zu setzen, während das Soll-Selbst das Setzen von negativ formulierten Ziele nach sich ziehen dürfte.

Ziele können auch als Leistungs- versus Lernziele formuliert werden (performance vs. learning goais; Dweck, 1996), als Leistungsversus Bewältigungsziele (performance vs. mastery goals; Ames \& Archer, 1988) oder ich- versus aufgabenbezogene Ziele (ego involvement vs. task involvement; Nicholls, 1979). Ziele im Leistungsbereich können entweder auf die Feststellung (Leistungsziele) oder das Wachstum der eigenen Fähigkeiten (Lernziele) ausgerichtet sein. Dweck (1996) beobachtete, daß implizite Theorien über das Wesen von Fähigkeiten die Wahl von Leistungs- versus Lernzielen bestimmen. Wenn eine Person Fähigkeiten als gegeben und 
unveränderbar ansieht (d. h. einer Entitätstheorie der Fähigkeit anhängt), präferiert sie Leistungsziele. Wenn eine Person dagegen davon ausgeht, daß Fähigkeiten durch Lernen verbessert werden können (d. h. einer Zuwachstheorie der Fähigkeit anhängt), setzt sie sich Lernziele. Analoge implizite Theorien über den Charakter einer Person oder ihre Fähigkeiten zu moralischem Handeln beeinflussen die Wahl von strafenden versus erklärenden Erziehungszielen.

Der Inhalt von gesetzten Zielen wird von Bedürfnissen, Wünschen und übergeordneten Zielen bestimmt. Nach Ryan, Sheldon, Kasser und Deci (1996) reflektieren Ziele die Bedürfnisse einer Person. Autonomie-, Kompetenz-, und soziale Integrationsbedürfnisse sollen das Setzen von Zielen fördern, die der Selbstverwirklichung dienen, und das Setzen von Zielen unterdrücken, die auf das materielle Fortkommen ausgerichtet sind. Markus und Nurius (1986) argumentieren, daß Personen nicht nur versuchen, sich so zu sehen wie sie sind (Selbstkonzept), sondern auch wie sie gerne sein möchten (mögliches Selbst oder possible self). Das mögliche Selbst liefert Ideen darüber, welche Ziele man sich für die Zukunft setzen könnte.

Wenn übergeordnete Ziele gesetzt worden sind (z. B. Arzt zu werden), bestimmen diese die Inhalte der untergeordneten Ziele. Die Inhalte übergeordneter "Be" Ziele determinieren die Inhalte untergeordneter "Do" Ziele, die wiederum die Inhalte entsprechender "motor-control" Ziele bestimmen (Carver \& Scheier, 1998, p. 72). "Be" Ziele werden in der Literatur auch als momentane Anliegen (current concerns; Klinger, 1977), ldentitätsziele (self-defining goals; Wicklund \& Goilwitzer, 1982), persönliche Projekte (personal projects; Little. 1983), persönliches Streben (personal strivings; Emmons, 1989), und non-normative Lebensaufgaben (life tasks; Cantor \& Fleeson, 1994) thematisiert. Während die Wahl übergeordneter "Be" Ziele primär von ihrer Wünschbarkeit und Machbarkeit abhängen dürfte, sollte die Wahl entsprechender untergeordneter "Do" Ziele auch von der Verbindlichkeit abhängen, mit der das zugehörige "Be" Ziel übernommen worden ist (Gollwitzer, 1987).

\section{Prozesse der Zielsetzung}

\section{Bewußte Prozesse}

Nachdem wir im vorigen dargestellt haben, welche Variablen die Wahl von Zielen mit bestimmten strukturellen und inhaltlichen Merkmalen bestimmen, wollen wir nun fragen, auf welchen Prozessen das Setzen von Zielen basiert. Nach Bandura (1997) fördert Erfolg im Erreichen von Zielen das Setzen von immer anspruchsvolieren Zielen, wobei die aufgrund der erfolgreichen Zielerreichung gestärkten Selbstwirksamkeitserwartungen die erneute, anspruchsvoilere Zielsetzung aus!ösen soll. Klinger sieht den zentraien Prozeß der Zieisetzung im Sich-verpflichten, einen bestimmten Anreiz zu erreichen (Klinger, 1977). Heckhausen und Kuhl (1985). argumentieren, daß das niedrigste Verbundenheitsgefühl zu einem Anreiz als Wunsch zum Ausdruck kommt. Um ein stärkeres Verbundenheitsgefühl zu erreichen, muß der Wunsch mehrere Prüfungen bestehen. Er soilte sich als machbar erweisen, die notwendigen Mittel und Gelegenheiten zu seiner Verwirklichung sollten vorhanden sein. Schließlich sollte er sich als wichtiger als andere Wünsche darstellen und seine Realisierung sollte dringlich sein. Erst wenn diese Prüfungen erfolgreich bestanden sind, wird der Wunsch zum Ziel.

Das Rubikonmodell der Handlungsphasen (Heckhausen \& Gollwitzer, 1987; Heckhausen, 1991; Gollwitzer, 1990) geht davon aus, daß Menschen immer mehr Wünsche besitzen als Zeit und Gelegenheit vorhanden sind, diese zu verwirklichen. Um zumindest einige Wünsche verwirklichen zu können, muß ausgewählt werden. Die Wahlkriterien sind dabei Wünschbarkeit und Machbarkeit. Wünsche mit hoher Wünschbarkeit und Machbarkeit werden am ehesten in Ziele transformiert. Die Transformation wird als eine (Auswahl-) Entscheidung verstanden, die ein Handlungsgefühl hervorruft. Die Entscheidung läßt den Wunsch als verbindliches Ziel erscheinen. Die Idee dieses Übergangs vom Wünschen zum Wollen wird im Rubikonmodell durch die Metapher des "Überschreitens des Rubikon" verdeutlicht. Nach dem Modell der Handlungsphasen (Gollwitzer. 1990; Heckhausen, 1991) setzt die Verwirklichung eines Wunsches die Lösung von vier aufeinanderfolgenden Aufgaben voraus: das Abwägen zwischen den verschiedenen Wünschen, damit ein geeigneter Wunsch ausgewählt werden kann (Vorentscheidungsphase); das Planen der Umsetzung des gewähiten Wunsches (der jetzt zum Ziel geworden ist), um mit dem Zielstreben beginnen zu können (Vorhandiungsphase); die Kontrolle oder Regulation zielgerichteten Handelns, damit der erwünschte Endzustand erreicht werden kann (Handlungsphase); und schließlich die Evaluation des bisher Erreichten im Vergleich zu dem, was ursprünglich gewünscht wurde, um das Zielstreben zu beenden oder fails nötig, erneut zu beginnen (Bewertungsphase). Man entscheidet sich, den Rubikon zu überschreiten (d. h. man bewegt sich vorwärts von der Vorentscheidungs- zur Vorhandlungsphase), wenn man erkennt, daß nicht nur die Wünschbarkeit und die Machbarkeit eines bestimmten Wunsches hoch genug sind, sondern auch die Abwägeaufgabe abgeschlossen ist (bzgl. Wünschbarkeit und Machbarkeit). Gollwitzer. Heckhausen und Ratajzak (1990) beobachteten, daß unentschlossene Personen tatsächlich eher dazu bereit waren, ihre Wünsche in Ziele zu verwandeln, wenn sie die Erfolgswahrscheinlichkeiten eingeschätzt hatten und sich zusätzlich ausführlich Gedanken über mögliche kurz- und langfristige, positive/negative Konsequenzen einer Zielrealisierung gemacht hatten. Unentschlossene Personen, die dazu verführt wurden, die Verwirklichung eines Wunsches zu planen (man sagte ihnen, sie möchten antizipierte Gelegenheiten mit intendiertem zielgerichteten Verhalten gedanklich verknüpfen), waren ebenfails bereiter, den Rubikon zu überschreiten. Offensichtlich sind unentschlossene Personen immer dann eher bereit, ihre Wünsche in Ziele zu verwandeln, wenn sie Anlaß zur Überzeugung haben, die Aufgabe des Abwägens von Wünschbarkeit und Machbarkeit sei abgeschlossen.

Die Theorie der Phantasierealisierung (Oettingen. 1996, 1997a) analysiert Zielsetzungsprozesse, indem sie zunächst zwei Formen des Zukunftsdenkens einander gegenüberstellt: Erwartungen versus freie Zukunftsphantasien. Erwartungen sind Urteile darüber, mit welcher Wahrscheinlichkeit ein bestimmtes Verhalten oder Ereignis auftreten wird. Sie basieren auf den in der Vergangenheit gemachten Erfahrungen und damit auf der Leistungsgeschichte einer Person. Zukunftsphantasien dagegen sind freie Gedanken und Vorstellungen über zukünftiges Verhalten oder Ereignisse, die vor dem geistigen Auge erscheinen, ganz unabhängig von deren Auftretenswahrscheinlichkeiten und damit von den in der Vergangenheit gezeigten Leistungen. Trotz niedriger Erfolgswahrscheinlichkeiten, bspw. einen Streit mit dem Partner zu lösen, kann eine Person in positiven Zukunftsphantasien über die erwünschte Harmonie schwelgen. 
Drei Wege zur Zielsetzung werden von der Theorie der Phantasierealisierung postuliert, die sich aus der Art und Weise ergeben, wie Personen mit ihren positiven Zukunftsphantasien gedanklich verfahren. Der erste Weg ist erwartungsgebunden, die beiden anderen dagegen lassen Erfolgserwartungen außer acht. Der erwartungsgebundene Weg resultiert aus der mentalen Kontrastierung positiver Zukunftsphantasien mit negativen Aspekten der widersprechenden Realität. Diese mentale Kontrastierung verankert freie Zukunftphantasien in der momentanen Realität. Folglich erscheint die positive Zukunft als etwas, das realisiert, und die widersprechende Realität als etwas, das verändert werden muß. Die dadurch erlebte Handlungsnotwendigkeit wirft die Frage auf, ob die Realität in Richtung Phantasie verändert werden kann. Die Antwort darauf liefern die von der Person eingeschätzten Erfolgswahrscheinlichkeiten oder Erfolgserwartungen, die Realität in Richtung Phantasie verändern zu können. Die mentale Kontrastierung der positiven Zukunft mit negativen Aspekten der widersprechenden Realität aktiviert somit die Erfolgserwartungen, die dann für die Beantwortung der Frage, ob man sich entsprechende Handiungsziele setzen soll, herangezogen werden können. Sind die Erfolgserwartungen hoch, kommt es zur hoch verbindlichen Zielsetzung, die Phantasie zu realisieren; sind sie dagegen niedrig, kommt es zu keiner Zielsetzung und man nimmt von einer Phantasierealisierung Abstand.

Der zweite Weg zur Zielrealisierung basiert auf dem bloßen Schwelgen in positiven Zukunftsphantasien, wobei die widersprechende Realität ignoriert wird. Die erwünschte Zukunft wird vor dem geistigen Auge spielend erreicht und genießerisch ausgekostet. Es wird somit keine Handlungsnotwendigkeit empfunden und relevante Erfolgswahrscheinlichkeiten werden nicht aktiviert. Zielsetzungen, die Phantasie zu realisieren, reflektieren folglich nicht die eingeschätzten Erfolgswahrscheinlichkeiten, sondern ausschließlich den Anreiz der in der Phantasie abgebildeten Ereignisse. Die Verbindlichkeit dieser Zie!setzungen ist deshalb genere!l moderat, was eine zu starke Verbindichkeit impliziert, wenn die Erfolgserwartungen hoch, und eine zu schwache Verbindlichkeit, wenn die Erfolgserwartungen niedrig sind.

Der dritte Weg ist das bioße Grübeln über die negativen Aspekte der widersprechenden Realität, wobei die positiven Zukunftsphantasien außer acht gelassen werden. Wiederum wird keine Handlungsnotwendigkeit erlebt, diesmal weil keine erwünschten Zukunftsereignisse dem potentiellen Handeln die Richtung weisen. Da deshalb keine Erwartungsurteile aktiviert werden, reflektiert die Verbindlichkeit relevanter Zielsetzungen ausschießlich den negativen Anreiz der widersprechenden Realität. Wie beim Schwelgen in positiven Zukunftsphantasien, fäll die Verbindlichkeit relevanter Zielsetzungen generell moderat aus, und damit zu stark, wenn die Erfolgsaussichten schlecht, und zu schwach, wenn diese gut sind.

Die Theorie der Phantasierealiserung wurde in einer Reihe von experimentellen Studien überprüft. In einer dieser Studien (Oettingen, in Druck a, Studie 1) wurde Studentinnen die Gelegenheit in Aussicht gestellt, einen attraktiven jungen Mann (einen Doktoranden am Institut, dessen Bild vorgelegt wurde) zu treffen. Zunächst mußte die Erfolgswahrscheinlichkeit angegeben werden, diese Person näher kennenzulernen. Danach listeten die Versuchspersonen mögliche positive Aspekte des Kennenlernens dieser Person auf (z. B. Freundschaft, gemeinsame Unternehmungen) sowie Aspekte der widersprechenden Realität ( $z$. B. die eigene Schüchternheit, das mögliche Desinteresse dieser Person). Schließlich wurden drei Gruppen gebildet, die die genannten Aspekte unterschiedlich gedanklich bearbeiten mußten. In der Phantasie-Realität-Kontrastierungsgruppe wurden sowohl die positiven Aspekte des Kennenlernens als auch die negativen Aspekte der widersprechenden Realität elaboriert, und zwar mit einem positiven Aspekt beginnend und dann immer abwechseind. In der bloßen positiven Phantasiegruppe wurden ausschließiich positive Aspekte des Kennenlernens elaboriert; in der bloßen negativen Realitätsgruppe ausschließlich negative Aspekte der widersprechenden Realität.

Die Verbindłichkeit relevanter Zielsetzungen (gemessen als Ungeduld, die attraktive Person kennenzulernen und als antizipiertes Ausmaß der Frustration, falls dies nicht gelingen solite) war in der Kontrastierungsgruppe eng mit den Erfolgserwartungen der Versuchspersonen verknüpft. In der bloßen Phantasie- und in der bloßen Realitätsgruppe standen Erfolgserwartungen und die Verbindlichkeit relevanter Zielsetzungen dagegen in keinem systematischen Zusammenhang. Ganz gleich ob die Erfolgserwartungen hoch oder niedrig waren, die Verbindlichkeit relevanter Zielsetzungen verharrte auf einem mittleren Niveau. Offensichtlich führt die mentale Kontrastierung von positiver Phantasie und negativer Realität zu verbindlichen Zielsetzungen, wenn die Erfolgsaussichten gut sind, und sie läßt von Zielsetzungen Abstand nehmen, wenn die Erfolgsaussichten schlecht sind. Das Schwelgen in positiven Phantasien sowie das Grübeln über die widersprechende negative Realität führen dagegen generell zu relativ schwachen Zielsetzungen, die ausschließlich vom positiven Anreiz der positiven Zukunft bzw. vom negativen Anreiz der widersprechenden Realität gestützt werden.

Ein weiteres Experiment (Oettingen, 2000, Studie 2), das mit kinderlosen Doktorandinnen durchgeführt wurde, analysierte das Entstehen der Zielsetzung, Familie und Beruf zu vereinbaren. Wiederum führte nur die mentale Kontrastierung von positiven Zukunftsphantasien mit der widersprechenden Realität dazu, daß Erfolgserwartungen die Verbindlichkeit relevanter Zielsetzungen (gemessen als antizipierte Frustration im Falle des Scheiterns, als Anstrengungsbereitschaft sowie als Planungsbereitschaft im Sinne der Durchführung sog. Prozeßsimulationen; Taylor. Pham, Rivkin \& Armor, 1998) bestimmten. Die Verbindlichkeit relevanter Zielsetzungen war dagegen bei den Versuchspersonen, die nur in positiven Zukunftsphantasien schwelgten oder nur über die negative Realität grübeiten erwartungsunabhängig.

In beiden Experimenten verhielten sich somit nur mental kontrastierende Personen rational in dem Sinne, daß die Höhe ihrer Erfolgserwartungen das Ausmaß der Verbindlichkeit relevanter Zielsetzungen bestimmte. Schwelgende und grübelnde Personen verhielten sich irrationai. Die Verbindlichkeit der Zielsetzungen war entweder zu hoch (wenn die Erfolgsaussichten schlecht waren) oder zu niedrig (wenn die Erfolgsaussichten gut waren). Eine Serie weiterer Experimente (Oettingen, 1999) konnte dieses Datenmuster replizieren, wobei es keinen Unterschied machte welche Phantasiethematiken (z. B. schulische Leistungen, soziale Konfliktlösung, emotionale und finanzielle Unabhängigkeit, Erfolg im Beruf) herangezogen und welche Versuchsparadigmen (d. h. Salienz- vs. Reinterpretationsmethoden) eingesetzt wurden, um die drei experimentellen Bedingungen (d. h. die mentale Kontrastierung, das Schwelgen in positiven Zukunitsphantasien, das Grübeln über die widersprechende Realität) herzustellen. Zusammengenommen sprechen die Ergebnisse dieser Studien dafür, daß die Art und Weise des Bedenkens einer erwünschten Zukunft bestimmt, ob es zu rationalen (erwartungsgebundenen) oder irrationalen (erwartungsungebundenen) Zielsetzungen kommt. 


\section{Automatische Prozesse}

Zieisetzungen beruhen nicht nur auf bewußten Überlegungen, sondern können auch aufgrund automatischer Prozesse zustandekommen. Nach Barghs (1990). Automotivtheorie können zwischen den kognitiven Repräsentationen von Zielen einerseits und den situativen Kontexten, in denen diesen Zielen in der Vergangenheit wiederholt und konsistent nachgegangen wurde, starke mentale Verknüpfungen entstehen. Dies führt dazu, daß Ziele automatisch aktiviert und verfolgt werden, wenn eine Person sich in den kritischen situativen Kontexten wiederfindet. Das automatisch aktivierte Ziel leitet das Verhalten der Person in der kritischen Situtation, ohne daß eine bewußte Zielsetzung erforderlich wäre. Die ursprünglich wichtige, bewußte Zielsetzung hat sich erübrigt.

Bargh. Gollwitzer, Lee-Chai und Barndollar (1999) prüften diese Annahme der direkten Zielaktivierung, indem sie versuchten herauszufinden, ob direkt aktivierte Ziele mit denselben Verhaltenskonsequenzen einhergehen wie bewußt gesetzte Ziele. In der Tat führten durch klassische, nicht-bewußte Primingverfahren aktivierte Leistungsziele zu besseren intellektuellen Leistungen als man sie bei Kontroilversuchspersonen beobachten konnte. Darüberhinaus bewirkten derartig aktivierte Zielsetzungen vergleichsweise erhöhte Persistenz bei der Aufgabenbearbeitung sowie eine erhöhte Wiederaufnahmerate. Mittels eines sog. Dissoziationsparadigmas wurde obendrein sichergestellt, daß die beobachteten Zieleffekte nicht durch die bloße Aktivierung (Priming) des semantischen Konzepts "Leistung" zustandegekommen waren.

Die von Bargh et al. beschriebenen automatischen Zielsetzungsprozesse basieren auf bewußten Zielsetzungen zu einem früheren Zeitpunkt. Die Automatisierung bezieht sich ausschließlich auf die situative Aktivierung einer in der Vergangenheit bewußt gesetzten Zielsetzung. Es erscheint jedoch durchaus möglich, daß zielgerichtetes Handeln nicht nur ohne bewußte Zielsetzungen im Hier und Jetzt, sondern auch ohne bewußte Zielsetzungen in der Vergangenheit zustandekommen kann. Wie bereits in der Einführung zu diesem Artikel ausgeführt, hat die behavioristische Forschung deutlich gemacht, daß bewußtes Zielsetzen für das Entstehen von Verhalten, das Merkmale des Zielstrebens trägt, nicht erforderlich ist. Derartiges Verhalten kann genauso gut durch operantes Konditionieren zustandekommen.

Die Überlegung, daß zielgerichtetes Verhalten völlig automatisch entstehen kann, wird auch durch rezente Forschung im Bereich der Bewegungskontrolle nahegelegt, die sich auf die dynamische Systemtheorie stützt (Kelso, 1995). Arbeiten in diesem Forschungsfeld sprechen dafür, daß zielgerichtetes Verhalten auch ohne die mentale Repräsentation von Zielen auskommen kann. Diese Annahme wird durch die Roboterforschung unterstützt (Brooks, 1991; Maes, 1994). Roboter können programmiert werden, komplexe zielgerichtete Verhaitensweisen auszuführen, ohne daß diese Programme auf Zielkonzepte zurückgreifen müssen. Konnektionistische Theorien stehen dem Zielkonzept ebenfalls kritisch gegenüber. Einige konnektionistische Theorien innerhalb der Psychologie verzichten ganz auf das Zielkonzept, während andere Prozesse der Zielsetzung durch die Entwicklung sog. parallel constraint satisfaction models (Read, Vanman; \& Miller, 1997) zu ersetzen versuchen.

Schließlich argumentieren Carver und Scheier (1999), daß es möglicherweise zwei Arten zielbezogener automatischer Prozesse gibt. Die erste Art wird in Barghs (1990) Automotivtheorie beschrieben und bezieht sich auf die Automatisierung durch wiederholtes und konsistentes Verknüpien eines bestimmten Zieis mit einer bestimmten Situation. Die zweite Art bezieht sich auf angeborene Verhaitenstendenzen, die nicht nur im Menschen, sondern auch in Organismen anderer Arten vorhanden sind. Carver und Scheier beschreiben diesen Typ von automatischen Prozessen als eine intuitive, wenig differenzierte "quick and dirty" Antwort auf die Anforderungen der Realität. Man braucht nicht zu warten bis eine bewußte Handiungsintention vorliegt, sondern handelt direkt. Ähnliches führen McClelland, Koestner und Weinberger (1989) aus, wenn sie Verhalten beschreiben, das auf sog. impliziten Motiven beruht. Implizite Motive sind biologische Bedürfnisse, die das Verhalten einer Person direkt durch angeborene Anreize steuern.

Wir haben die verschiedenen Quellen automatischen zielgerichteten Verhaltens an dieser Stelle erwähnt, um dem Leser bewußt zu machen, daß Verhalten, das Merkmale der Zielgerichtetheit trägt, nicht notwendigerweise einer bewußten Zieisetzung bedarf oder der Aktivierung der mentalen Repräsentation eines in der Vergangenheit bewußt gesetzten Ziels. Auch wenn einige der letztgenannten Theorien die Existenz und Relevanz bewußter Zielsetzungen oder mentaler Repräsentationen von Zielen in Frage stellen, so scheint uns die anspruchsvoliere Forschungsfrage der nächsten Jahre die zu sein, wie automatische und bewußte Zielsetzungsprozesse zusammenwirken.

\section{Zielstreben}

\section{Determinanten des Zielstrebens}

Ziele variieren neben ihren inhalten auch in ihren struktureilen Merkmalen. Sie können herausfordernd oder anspruchslos sein, spezifisch oder vage, proximal oder distal, positiv oder negativ formuliert, etc., wobei sowohl die Zielinhalte als auch die strukturellen Merkmale den Erfolg des Zielstrebens beeinflussen.

\section{Strukturelle Merkmale}

Sehr anspruchsvolle spezifisch formulierte Ziele begünstigen den Erfolg stärker als moderat anspruchsvolle spezifische Ziele und stärker als sehr anspruchsvolle vage Ziele - vorausgesetzt, daß Leistungsrückmeldungen häufig gegeben werden, eine starke Zielverpflichtung vorliegt, das Ziel nicht zu komplex ist, und mangelnde Kompetenz oder die Abwesenheit von Gelegenheiten die Zielverwirklichung nicht vereiteln (Locke \& Latham, 1990). Irrelevant dagegen scheint zu sein, ob die Ziele von anderen aufgetragen, von der Person selbst oder gemeinsam mit anderen gesetzt wurden. Als potentielie Mediatoren der Zielspezifitätseffekte nennen Locke und Latham (1990) erhöhte Persistenz und die Aufmerksamkeitslenkung auf zielgerichtetes Verhalten sowie die Bereitschaft, 
die Zielverwirklichung zu planen und Rückmeldung anzunehmen.

Als weitere struktureile Merkmale von Zielen werden Zeitperspektive, Ergebnisfokus (d. h. ob sie positiv oder negativ formuliert werden) sowie Leistungs- versus Lernorientierung genannt. Bandura und Schunk (1981) unterscheiden zwei Zeitperspektiven der Zielerreichung, proximal und distal. Proximale Ziele beziehen sich auf die nahe Zukunft, während distale Ziele in die ferne Zukunft weisen. In einem Experiment legten Bandura und Schunk an Mathematik uninteressierten, leistungsschwachen Kindern ein selbstgesteuertes Lernprogramm vor. Eine Versuchsgruppe erhielt ein distales Ziel (42 Seiten in 7 Sitzungen), die andere ein distales plus ein proximales Subziel (6 Seiten pro Sitzung in 7 Sitzungen). Die zusätzlichen proximalen Ziele verbesserten die distales plus ein proximales Subziel (6 Seiten pro Sitzung in 7 Sitzungen). Die zusatzich vermehrte Leistungsrückmeldung erzielte Erfoig dürtte sich jedoch bei Zieien, die auf eine Hemmung von Verhalten ( $z$. B. nicht mehr zu rauchen) ausgerichtet sind, in Mißerfolg verkehren, denn hier sind Mißerfolge salienter und verieiten so eher zum Aufgeben. Tatsächlich berichten Cochran und Tesser (1996), daß sich die berichteten Vorteile proximaler Ziele für mißerfolgsvermeidende Ziele genau umkehren.

Nach Higgins (1997) werden positiv formulierte Ziele dann am ehesten erreicht, wenn sowohl die Erfolgserwartungen als auch der eingeschätzte Anreiz am höchsten sind; bei negativ formulierten Zielen ist dieser Effekt dagegen weniger ausgeprägt. Wenn sehr wünschenswerte und machbare Wünsche in Ziele verwandelt werden, scheint es damit angebracht, diese positiv zu formulieren. Positiv formulierte Ziele konzeptualisieren Erfolg als etwas, was herbeigeführt werden muß, negativ formulierte Ziele dagegen als einen Erhalt des Status quo.

Ob Ziele als Leistungs- oder Lernziele begriffen werden, beeinflußt ebenfalis den Erfoig im Zielstreben (Dweck. 1996). Leistungsziele führen zu weniger Erfolg als Lernziele, weil Leistungsziele die Mißerfolgsverarbeitung erschweren. Für Personen mit Leistungszielen signalisiert Mißerfolg Mangel an Fähigkeit und kann daher zum Aufgeben führen. Personen mit Lernzielen betrachten Mißerfolg dagegen als Hinweis darauf, daß neue Handilungsstrategien entwickelt und erprobt werden müssen. Ihr Verhalten richtet sich demnach an der Beseitigung von Mißerfolgsursachen aus, was letztlich dem Erfolg beim Zieistreben zugutekommt. Elliot und Church (1997) beobachteten, daß Leistungsziele jedoch weniger problematisch sind, wenn sie als Annäherungsziele (z. B. ich möchte gute Noten erreichen) als wenn sie als Vermeidungziele (z. B. ich möchte schlechte Noten vermeiden) gesetzt werden.

\section{Inhaltliche Merkmale}

Ryan, Sheldon, Kasser und Deci (1996) vermuten, daß auf Autonomie und Kompetenz ausgerichtete Ziele sowie Ziele sozialer Integration, Kreativität und kognitiver Flexibilität, die Verarbeitung relevanter Informationen und die Mißerfolgsbewältigung begünstigen. Mediator dieser Effekte ist die intrinsische Selbstregulation, die durch die Bedürfnisse nach Autonomie, Kompetenz und sozialer Integration angeregt wird. Dieses konstruktive Zielstreben wird einem extrinsischen Zielstreben gegenübergestelit, wobei letzteres von äußeren (z. B. Autoritäten) oder von inneren (z. B. Schuldgefühlen) Einflüssen gesteuert wird. Ryan et al. (1996). berichten auch Nebeneffekte des Zielstrebens. Ziele, die auf Autonomie, Kompetenz und soziale Integration ausgerichtet sind, fördern subjektives Wohlbefinden und Lebenszufriedenheit. Personen, die sich Ziele gesetzt hatten, wie bspw. viel Geld zu verdienen, berühmt zu werden, oder hohen Status zu erlangen, weisen ein geringeres subjektives Wohlbefinden auf als Personen, deren Ziele auf Freundschaft und soziale Verantwortung ausgerichtet sind (Kasser \& Ryan, 1993).

Subjektives Wohibefinden wurde auch in anderen Zielinhaltsbereichen untersucht. Nach Emmons (1996) ist der Anteil von Intimitätszielen an der Gesamtzielanzahl ein positiver Prädiktor, während der Anteil an Leistungs- und Machtzielen sich negativ auswirkt. Brunstein, Schultheiss und Maier (1999) geben jedoch zu bedenken, daß Merkmale von Zielen das Wohlbefinden nur unter bestimmten Bedingungen beeinflussen. Beispielsweise fördern verbindlich gesetzte Intimitätsziele nur dann die Lebenszufriedenheit, wenn sie als machbar erscheinen; wenn die Machbarkeit niedrig ist, reduziert die verbindliche Übernahme derartiger Ziele die Lebenszufriedenheit. Auch sind Intimitätsziele nur dann von Vorteil, wenn gleichzeitig soziale Unterstützung seitens wichtiger Bezugspersonen vorliegt. Schließlich hängen die Effekte von Zielsetzungen auf emotionales Wohlbefinden davon ab, inwieweit die Bedürfnisse oder impliziten Motive (McClelland, 1985) einer Person mit den gesetzten Zielen übereinstimmen. Personen mit starken Leistungs- und Machtbedürfnissen, die sich Ziele gleicher Thematik setzen - wie auch Personen mit starken Affiliations- und Intimitätsbedürfnissen und gleichthematischen Zielen - berichten über besseres emotionales Wohlbefinden als Personen, bei denen Bedürfnisse und Ziele nicht gleichthematisch sind.

\section{Prozesse des Zielstrebens}

Der Weg von der Zielsetzung zur Zielerreichung ist oft lang und beschwerlich. Zielsetzung ist nur der erste Schritt zum Erfolg, der eine Vielzahl noch zu iösender Aufgaben nach sich zieht. Im vorhergehenden Abschnitt haben wir diskutiert, inwieweit verschiedene strukturelle und thematische Zielmerkmale Erfolg im Zielstreben vorhersagen. Die prozessorientierte Forschungsperspektive fokussiert dagegen auf die Frage, wie die verschiedenen Pobleme, die sich einem erfolgreichen Zielstreben in den Weg stellen, effektiv gelöst werden können. Um diese Probleme, die die Handlungsinitiierung einerseits und die Zielvoilendung andererseits betreffen, effektiv lösen zu können, muß eine Person die sich bietenden Gelegenheiten zum Handeln wahrnehmen, sich vor Ablenkungen abschirmen, die Stärke aufgebrachter Anstrengungen den aufkommenden Schwierigkeiten flexibel anpassen, Hindernisse umschiffen, Mißerfolge verarbeiten und Schwächen kompensieren sowie Zielkonflikte auflösen. Wie eine Person all diese Probleme der Zielrealisierung bewältigen kann, ist Thema einer Reihe von Theorien, die im folgenden erörtert werden.

\section{Planende Bewußtseinslage}

Das Modell der Handlungsphasen (Heckhausen \& Gollwitzer, 1987; Heckhausen, 1991) definiert erfolgreiches Zielstreben als das 
Lösen einer Reihe von aufeinanderfoigenden Aufgaben: Das Abwägen von Wünschen (potentiellen Zieien) und die Auswahl
bestimmter Wünsche, das Planen zielgerichteten Handelns und die Handiungsinitiierung, das erfoigreiche Abschließen
zielgerichteten Handelns und schließlich die Bewertung der erzielten Handlungsergebnisse und -konsequenzen. Das
Aufgabenkonzept impliziert, daß Personen ihr Zielstreben fördern können, indem sie die entsprechenden kognitiven
Bewußtseinslagen entwickeln, die das Lösen der genannten Aufgaben erleichtern (Gollwitzer, 1990).

Experimentefle Studien zu den Bewußtseinslagen, die mit dem Abwägen von Wünschen (abwägende Bewußtseinslage) oder mit dem Planen der Durchführung zieigerichteter Handlungen (planende Bewußtseinslage) einhergehen, unterstützen diese Überlegungen (Gollwitzer \& Bayer, 1999). Wenn Personen aufgefordert werden, die Realisierung eines gesetzten Ziels im Detail zu planen, entsteht eine planende Bewußtseinslage, die folgende Merkmale trägt: Personen verschließen sich gegenüber nichtzieirelevanten Informationen, während sie gegenüber für die Zielverwirklichung reievanten Informationen offener werden. Informationen, die sich auf die Wünschbarkeit des gesetzten Ziels beziehen, werden parteilsch bearbeitet in dem Sinne, daß positive Argumente stärker berücksichtigt werden als negative. Informationen, die sich auf die Machbarkeit beziehen, werden iliusionär optimistisch interpretiert. Dieser Optimismus fördert Kontrollillusionen angesichts unkontrollierbarer Ereignisse, und kann sogar bei depressiven Personen beobachtet werden. Die Selbstwahrnehmung wichtiger persönlicher Eigenschaften (z. B. Fröhlichkeit, Intelligenz, soziale Sensitivität) wird gestärkt, während die wahrgenomme Vulnerabilität sowohl bzgl. kontroliierbarer als auch nicht kontrollierbarer Risiken geschwächt wird ( $z$. B. eine Arzneimittelsucht entwickeln bzw. den Partner verlieren). Die planende kewtrolierbarer Risiken geschwäseinslage begünstigt die Zielerreichung, indem sie die Bewältigung einiger für das Zielstreben typischer Probleme erleichtert, wie z. B. abgelenkt werden oder Zweifel an der Wünschbarkeit und Machbarkeit des gewählten Ziels hegen.

\section{Planen durch Vorsatzbildung}

Ziele verpflichten eine Person auf das Erreichen einer erwünschten Zukunft, aber nicht auf das Wann, Wo und Wie der Ausführung zielgerichteten Handelns. Solche zusätzlichen Verpflichtungen können dadurch eingegangen werden, daß man das Zieistreben durch Vorsätze in der Form "sobald die Situation x auftritt, will ich das zielgerichtete Verhalten y ausführen!" plant. Gollwitzer (1993) postuliert, daß Vorsätze eine sehr wirkungsvolle selbstregulatorische Strategie darstellen, die das Überwinden von Handlungsinitierungsproblemen erleichtert (z. B. wenn man günstige Gelegenheiten zum Handeln übersieht, weil man müde, in anderen Aktivitäten absorbiert oder in seinen Gedanken verloren ist). In einer Vielzahl von Studien (Gollwitzer, 1999) konnte gezeigt werden, daß schwierig zu erreichende Ziele durch das Fassen von Vorsätzen entscheidend begünstigt werden. Dieser Effekt konnte bei inhaltlich ganz verschiedenen Zie!projekten verläßlich beobachtet werden, wie z. B. einen interpersonellen Konflikt zu lösen, eine medizinische Selbstuntersuchung durchzuführen, ein Vitaminpräparat regelmäßig einzunehmen, gesund zu essen oder sich sportlich zu betätigen. Der Vorsatzeffekt läßt sich selbst bei Personen beobachten, die bekannt dafür sind, daß sie Schwierigkeiten mit der Umsetzung von Zielen in Handeln haben, wie z. B. Opiatabhängige im Entzug und schizophrene Patienten.

Da Vorsätze Verknüpfungen zwischen antizipierten situationalen Stimuli und zielgerichtetem Verhalten spezifizieren, wird angenommen (Goilwitzer, 1993), daß Vorsätze die Kontrolle des Handelns vom Selbst weg an die Umwelt delegieren und auf diese Weise die Handlungsinitiierung erleichtern. Die mentale Repräsentation der situativen Stimuli erfährt eine erhöhte Aktivierung, die diese Stimúfi leichter zugänglich macht. Verschiedene Experimente (Gollwitzer, 1999) machen deutlich, daß die in Vorsätzen spezifizierten situativen Stimuli (Gelegenheiten) im Vergleich zu nicht-spezifizierten Gelegenheiten leicher entdeckt und erinnert werden, sowie von sich aus Aufmerksamkeit auf sich ziehen. Weiterhin werden durch Vorsätze starke assoziative Verknüpfungen zwischen der mentalen Repräsentation situativer Stimuli einerseits und zielgerichtetem Verhalten andererseits geschaffen, wie sie sonst nur durch konsistentes und wiederholtes gemeinsames Auftreten entstehen. Folglich wird die Handlungsinitiierung automatisiert. Verschiedene Experimente zeigen, daß das in Vorsätzen spezifizierte zielgerichtete Verhalten unmittelbar und anstrengungsfrei initiiert wird, sobald die kritische Situation vorliegt. Weiterhin genügt bereits die subliminale Repräsentation der kritischen Situation, um kognitive Konzepte zu aktivieren, die der Ausführung des intendierten Verhaltens dienen. Schließlich profitieren selbst Patienten mit einer Frontalhirnverletzung (die dafür bekannt sind, Schwierigkeiten bei der bewußten Kontrolle ihres Handelns zu haben, während die Ausführung automatischen Verhaltens nicht beeinträchtigt ist) von der Vorsatzbildung.

Vorsätze reduzieren nicht nur Probleme bei der Handlungsinitiierung, sondern auch Probleme, die mit der erfolgreichen Erledigung initiierter Handlungen zu tun haben (Gollwitzer \& Schaal, 1998). Sie schaffen einen wirksamen Schutz gegen attraktive Ablenkungen, und dies selbst dann, wenn man mühsame zielgerichtete Handlungen ausführt, wie z.B. das Bearbeiten von Rechenaufgaben. Auch Zielsetzungen, die sich auf das Unterdrücken von unerwünschten Gewohnheitshandlungen beziehen ( $z$. B. Stereotypisierungen von und Vorurteile gegenüber Randgruppenmitgliedern) werden erfolgreicher umgesetzt, wenn sie mit Vorsätzen ausgestattet werden. Schließlich schützen Vorsätze vor unerwünschten Einflüssen direkt aktivierter Zielsetzungen im Sinne Barghs (1990). Man kann sich auf diese kritischen Situationen durch das Fassen antagonistischer Ziele vorbereiten, und wenn letztere obendrein mit Vorsätzen ausgestattet werden, unterdrücken sie erfoigreich das direkt aktivierte, unerwünschte Zielstreben (Goliwitzer, 1999). Zusammenfassend gilt, daß Vorsätze eine Art der automatischen Ausiösung von Verhalten produzieren, die keiner konsistenten und wiederholten Übung bedarf. Vielmehr delegieren Personen die Kontrolle zielgerichteten Verhaltens an antizipierte kritische situationale Stimuli. Diese leicht zu erwerbende selbst-regulatorische Strategie kann dazu eingesetzt werden, die Initijerung zielgerichteten Verhaltens entschieden voranzutreiben. Gieichzeitig erhöht diese Strategie jedoch die Handilungsflexibilität insofern als man dadurch unerwünschten automatischen Denk-, Füh!- und Verhaltensmustern leichter entkommen kann.

Es gibt neben der Vorsatzbildung auch andere Formen effektiven Planens, wie z. B. mentale Simulationen, die verschiedene Wege zur Zielerreichung in Gedanken erproben. Taylor et al. (1998) nennen derartige mentale Simulationen Prozeßsimulationen (process simulations). Wenn diese wiederholt ausgeführt werden, fördern sie die Zielrealisierung, wie z. B. das Erreichen von guten Noten in Klausuren. Offensichtlich resultiert das Planen in Form des wiederholten mentaien Simulierens des Wegs zum Ziel ebenfalis in wirkungsvollen Plänen. 


\section{Handiungs- und Lageorientierung}

Konfligierendem Zielstreben wird in Kuhls Theorie der Handlungskontrolle besondere Aufmerksamkeit geschenkt (im Überblick, Kuhi \& Beckmann, 1994). Für den erfolgreichen Ablauf einer Handlungssequenz muß man ein momentan aktives Ziel von konfligierenden, alternativen Zielen abschirmen. Kuhl nennt diesen Abschirmmechanismus Handlungskontrolle und unterscheidet eine Reihe verschiedener Kontrollstrategien, wie $z$. B. Aufmerksamkeitskontrolle, Emotionskontroile und Umweltkontrolle. Durch Umweltkontrolle bspw. verhindert man, daß ein im Moment erfolgreich ablaufendes Zieistreben aus dem Ruder gerät, indem man potentielle Ablenkungen vorsichtshalber von vornherein aus der Handiungssituation entfernt.

$\mathrm{Ob}$ und wie effektiv Kontrollstrategien eingesetzt werden, hängt vom Kontrollmodus der Person ab. Eine handlungsorientierte Person konzentriert sich auf das Planen und Initiieren zielgerichteten Verhaltens, reagiert flexibel auf die situativen Anforderungen und nützt die bekannten Kontrollstrategien. Eine lageorientierte Person dagegen kann sich nicht von unerledigten alten Zieien ablösen, ruminiert über erlittene Mißerfolge und veriiert sich in Gedanken über zukünftige Erfolge. Handlungs- und Lageorientierung kennt situative Auslöser ( $z$. B. ein überraschendes Ereignis bzw. persistenter Mißerfolg), ist jedoch in einer persönlichen Disposition begründet.

Rezente experimentelle Forschung zur Lageorientierung hat eine weitere volitionale Schwäche entdeckt. Lageorientierte Personen verkennen von anderen aufgetragene Ziele ais selbstgesetzte Ziele. Dieser Befund hat die Entwicklung einer neuen theoretischen Perspektive (Kuhl,2000) stimuliert, die óle volitionale Kontrolle von Handlungen als das Endergebnis des Zusammenwirkens einer Reihe verschiedener mentaler Subsysteme versteht (d. h. dem Absichtsgedächtnis, dem Extensionsgedächtnis, der intuitiven Handlungskontrolle und dem Objekterkennen). Handlungs- versus Lageorientierung werden dabei als Parameter verstanden, die das Zusammenwirken dieser Systeme modulieren, was schließlich zu einer unterschiedlichen Art der Handlungskontrolle mit jeweils anderen Konsequenzen führt.

\section{Die Wiederaufnahme unterbrochenen Zielstrebens}

Übergeordnete Ziele (z. B. eine beliebte Person zu werden) bieten verschiedene alternative Wege der Zielrealisierung an. Falls einer dieser Wege blockiert ist, kann man versuchen, das Ziel auf andere Weise zu erreichen. Die Selbstergänzungstheorie (Wicklund \& Gollwitzer 1982) analysiert diese Frage der Kompensation hinsichtlich sog. Identitätsziele. Diese Ziele spezifizieren eine bestimmte ldentität, wie z.B. Wissenschaftler, Mutter, oder liberale Person, als erwünschten zukünftigen Zustand. Da viele verschiedene Dinge den Besitz solcher Identitäten anzeigen, bedeutet das Streben nach diesen Identitäten nichts anderes als das Ansammeln von relevanten Indikatoren (oder Symbolen). Solche Indikatoren reichen von relevanten materiellen Symbolen ( $z$. B. Bücher und Auszeichnungen) zu relevanten Selbstbeschreibungen (z. B. der Gebrauch von Titein) und tatsächlichen Leistungen (z. B. die Durchführung wichtiger Forschungsarbeiten). Die Selbstergänzungstheorie postuliert, daß bei Ausfällen bzgl. eines Indikators ein Unvolikkommenheitsgefühl entsteht, das kompensatorische Selbstsymbolisierungen anregt. Letztere bestehen darin, daß man auf den Besitz alternativer Indikatoren verweist oder versucht, alternative Indikatoren zu erwerben.

Dieses Kompensationsprinzip hat sich bzgl. des Strebens nach verschiedenen Identitätszielen und auch bzgl. verschiedener Arten von Indikatoren empirisch bestätigen lassen (Gollwitzer \& Kirchhof, 1998). Leicht zugängliche Indikatoren (z. B.

Selbstbeschreibungen) stellten sich dabei als wirksamer Ersatz für Indikatoren heraus, die nicht so leicht zugänglich sind (z. B. relevante Leistungen). Anfänger in einem bestimmten ldentitätsbereich ( $z$. B. Wissenschaft) können somit bereits den Besitz der erwünschten Identität symbolisieren, obwohl es auf der Ebene der erzielten relevanten Leistungen noch wenig vorzuweisen gibt. Die Forschung zu Selbstergänzungsprozessen hat weiterhin entdeckt, daß effektives Selbstsymbolisieren einer sozialen Realität bedarf. Kompensatorische Anstrengungen sind immer dann besonders wirksam (im Sinne des Produzierens symbolischer Vollkommenheit), wenn andere Personen davon Kenntnis nehmen. Dies impliziert jedoch Kosten. Kompensierende Personen betrachten andere nur als potentielle Kenntnisnehmer und besitzen deshalb nur eine geringe Sensibilität hinsichtlich der tatsächlichen Interessen anderer. Auch führt das Öffentlichmachen von intentionen, einen bestimmten identitätsbezogenen Indikator erwerben zu wollen ( $z$. B. fleißig zu studieren für einen Jurastudenten) dazu, daß die tatsächlichen Anstrengungen gering ausfallen, weil die Kenntnisgabe der Intention bereits ein Gefühl der Vollkommenheit (als Jurist) induziert (Gollwitzer, Bayer, Scherer \& Seifert, 1999).

Die Selbstergänzungstheorie mag den Leser an die Selbstaffirmationstheorie von Steele (1988) erinnern. Aber die Selbstergänzungstheorie ist eine Zieltheorie und keine Selbstwertheorie (kritisch dazu, Tesser, Martin \& Cornell, 1996). Nach Steele stärkt alles, was ein gutes Gefühl bzgl, der eigenen Person aufkommen läßt, einen wie auch immer geschwächten Seibstwert. Die Selbstergänzungstheorie postuliert dagegen, daß identitätsbezogene Unvollkommenheit nur durch den Verweis auf oder Erwerb von alternativen ldentitätssymbolen kompensiert werden kann. Rezente Befunde machen deutlich, daß das bloße Stützen des Seibstwertes keine identitätsbezogene Volikommenheit erzeugt (Gollwitzer et al.. 1999).

\section{Anstrengungsregulation}

Man kann die Realisierung von Zielen sicherstellen, indem man für erlittene Mißerfolge kompensiert, aber man kann genauso gut versuchen, Mißerfolge durch erhöhte Anstrengung erst gar nicht eintreten zu lassen. Das Vermeiden von Mißerfolgen wird dann besonders wichtig, wenn Schwierigkeiten auftreten. Brehm und Wright (Brehm \& Self, 1989; Wright, 1996) haben eine Theorie der Energetisierung vorgestellt, wonach die Anstrengungsbereitschaft direkt von der wahrgenommenen Schwierigkeit der Aufgabe abhängt. Mit dem Ansteigen der Schwierigkeit steigt auch die aufgebrachte Anstrengung, es sei denn, die anstehende Aufgabe wird als unlösbar wahrgenommen. Die Anstrengungssteigerung wird jedoch noch durch einen anderen Faktor limitiert: die potentielle Motivation, die aus bedürfnișbezogenen Variablen resultiert (d. h. die Stärke bedürfnisbezogener oder übergeordneter Ziele, der Anreizwert des Aufgabenziels und die Instrumentalität der Lösung der Aufgabe für die Bedürfnisbefriedigung oder das Erreichen des übergeordneten Ziels). Wenn die potentielle Motivation niedrig ist, lohnt es nicht, die Anstrengung zu steigern, wenn eine zunächst 
einfache Aufgabe schwieriger wird, denn die obere Grenze der Anstrengungssteigerung ist hier niedrig und schnell erreicht. Ist die potentielle Motivation dagegen hoch, wird ein Ansteigen der Schwierigkeit mit mehr Anstrengung beantwortet und dies bis zu sehr hohen Schwierigkeitsniveaus. Die obere Grenze der Anstrengungssteigerung ist hoch und wird erst erreicht, wenn bereits sehr viel Anstrengung aufgebracht wurde.

Zur Überprüfung der Theorie wurde die potentielle Motivation entweder dadurch variiert, daß man hohe oder niedrige Belohnungen für die erfolgreiche Aufgabenbearbeitung aussetzte oder eine hohe Belohnung mehr oder weniger wahrscheinlich machte. Die Anstrengungssteigerung wurde meist durch kardio-vaskuläre Reaktionen (d. h. Herzschlagrate und systolischer Blutdruck) erfaßt. Tatsächlich begrenzt potentieile Motivation die lineare Beziehung zwischen Aufgabenschwierigkeit und Anstrengung nach oben hin. Rezente Forschung zieht die Energetisierungstheorie heran, um die bekannten Unterschiede zwischen Männern und Frauen bzgl. der Anstrengungsbereitschaft bei der Bearbeitung geschlechtstypischer Aufgaben sowie die Effekte von öffentlichen und privaten Arbeitsbedingungen auf die Anstrengungsbereitschaft zu verstehen (Wright et al. 1995, 1997).

\section{Diskrepanzreduktion}

In den bereits dargestellten Theorien des Zielstrebens werden Ziele explizit oder zumindest implizit als etwas Attraktives verstanden, das die Person zu erreichen wünscht. Ziele sind nicht einfach "kalte" mentale Repräsentationen, die ausschließlich einen Standard oder Referenzwert spezifizieren, sondern kognitiv explizierte und elaborierte Anreize. Derartige motivationale Zieltheorien stehen in Konkurrenz zu betont kognitiven Ansätzen, wonach Ziele nur Leistungsstandards spezifizieren. Nach Bandura (1997) haben Ziele per se keine motivationalen Konsequenzen. Sie spezifizieren nur die Bedingungen, die eine positive oder negative Selbstbewertung erlauben. Wird ein gesetztes Ziel erreicht, erlaubt dies eine positive Selbstbewertung, während jedes Nichterreichen des Ziels zu einer negativen Seibstbewertung führen muß. Die Person wird von der Vermeidung der negativen Selbstbewertung, die mit Zieldiskrepanzen assozilert ist, vorwärts geschoben, und von der antizipierten positiven Selbstbewertung, die mit dem Schließen der Diskrepanz verknüpft ist, vorwärts gezogen. Demnach soltten Ziele Anstrengungen zur Zielerreichung nur dann auslösen, wenn eine Diskrepanz zwischen dem Status quo und dem gesetzten Ziel bemerkt wird. Bandura schlägt deshalb das häutige Geben von Rückmeldungen als wirksame Maßnahme zur Stimulierung des Zielstrebens vor. Personen dürften jedoch nach Bandura (1997) nur dann versuchen, die Diskrepanz zu reduzieren, wenn sie ein hohes Selbstwirksamkeitsgefüh! bzgl. der Ausführung zielrealisierender Handlungen besitzen.

Carver und Scheier (1998) entwickelten eine andere Diskrepanztheorie des Zielstrebens. Angeregt durch kybernetische Kontroiltheorien nutzen sie als zentrales Konzept ihrer Analyse die negative Rückmeldeschieife. Carver und Scheiers Analyse streicht vor allem die hierarchische Struktur des Zielstrebens heraus, indem Kaskaden von negativen Rückmeldeschleifen postuliert werden. Zielgerichtetes Verhalten wird auf der mittleren Ebene ("Do"-Ziele) reguliert, und erst wenn die Person selbstaufmerksam wird, bewegt sich die Regulation auf eine höhere Ebene ("Be"-Ziele). Das Bemerken von Diskrepanzen auf der "Be"-Ebene oder der "Do"-Ebene verschiebt die Zielregulation auf die jeweils niedrigere Zielebene bzw. gleich auf das Verhalten, das die Diskrepanz reduzieren soll. Diskrepanzen werden jedoch nur dann reduziert, wenn die Ergebniserwartungen hoch sind. Eine positive Reaktion als Konsequenz der Zielerreichung wird nicht postuliert und auch das Bemerken der Diskrepanz löst keine negativen Gefühle aus.Vielmehr wird die Quelle positiver und negativer Gefühle beim Zielstreben in der Geschwindigkeit der Diskrepanzreduktion vermutet. Die Qualität dieser Gefühle wird wiederum durch eine negative Rückmeldeschleife reguliert. Liegt die Geschwindigkeit über dem gesetzten Standard, entstehen positive Gefühle, liegt sie darunter, ergeben sich negative Gefühle.

Die genannten Diskrepanztheorien konzeptualisieren Ziele als "kalte" mentale Repräsentationen von Leistungsstandards ohne Bezug zu Bedürfnissen und Anreizen. Diese Ansätze können nicht erklären, warum Motivation (siehe z. B. Brehm und Wrights Konzept der potentiellen Motivation) die Relation zwischen Aufgabenschwierigkeit und Anstrengung moderiert. Obendrein sollte nach den genannten Diskrepanztheorien ein Anstieg der Aufgabenschwierigkeit die Anstrengungsbereitschaft reduzieren, weil jede bemerkte Steigerung der Aufgabenschwierigkeit die Selbstwirksamkeitsgefühle und die Ergebniserwartung reduzieren dürfte. Wie Brehm und Wright jedoch wiederholt demonstrieren konnten, führt hohe potentieile Motivation dazu, daß Personen ihre Anstrengungen steigern, sobald erhöhte Aufgabenschwierigkeit die Zielerreichung bedroht. Schließlich impliziert Carver und Scheiers Konzeptualisierung der Regulation der Geschwindigkeit der Diskrepanzreduktion, daß positive Diskrepanzen (d. h. sich auf das Ziel hin zu schnell bewegen) genauso bereitwillig reduziert werden wie negative Diskrepanzen (d. h. sich auf das Ziel hin zu langsam bewegen). Aus motivationaler Perspektive, wonach Ziele einen erwünschten Zustand repräsentieren, soliten Personen jedoch weniger bereit sein, positive Diskrepanzen zu reduzieren als negative Diskrepanzen (Goliwitzer \& Rohloff. 1999).

\section{Rück- und Ausblick}

Obwohl die theoretische und empirische Analyse der Bedingungen und Prozesse des Zielsetzens und Zielstrebens in den letzten 10 Jahren an Bedeutung gewonnen hat, gibt es noch eine Vielzahi von zielbezogenen Phänomenen, die bislang ein Schattendasein geführt haben. Eines der weitgehend ununtersuchten Phänomene ist das der Zielkonflikte. Es ist eine offene Frage, wie es dazu kommt, daß eine Person konfligierende Ziele unterhält. Die Analyse der Frage, wann und wie positive Zukunftsphantasien entstehen, könnte hier von Nutzen sein. Zukunftsphantasien sind zunächst ein Produkt des kulturellen Kontextes einer Person und der Bedürfnisse, Werte, Einsteilungen und Interessen, die sie in diesem Rahmen entwickelt. Ob eine Person schließlich ihre Zukunftsphantasien in verbindliche Zielsetzungen transformiert, solite davon abhängen, ob die Person bereit ist, ihre Zukunftsphantasien mit der widersprechenden Gegenwart mental zu kontrastieren. Diese Form des selbstregulatorischen Denkens mag wiederum kulturelle Wurzeln haben (Oettingen, $1997 \mathrm{~b}$ ).

Wenn gesetzte Ziele bereits in Konflikt geraten sind, müssen diese Konflikte gelöst werden. Emmons (1996) verweist auf die Möglichkeit kreativer Integrationen, wobei neue Ziele gesetzt werden, die die beiden konfligierenden Ziele in gleicher Weise 
berücksichtigen (z. B. wenn Leistungs- und Affiliationsziele durch die Übernahme sozialer Verantwortung miteinander versöhnt werden). Cantor und Fleeson (1994) argumentieren darüber hinaus, daß übergeordnete Ziele (z. B. das Studium abschließen) nur dann erreicht werden können, wenn untergeordnete Ziele, die miteinander in Konflikt stehen, strategisch miteinander verknüpft werden (z. B. wenn der Konflikt zwischen sich auf das Examen vorbereiten vs. mit anderen zusammen sein wollen durch die Teilnahme an Arbeitsgruppen aufgelöst wird). Aber häufig können Zielkonflikte nur beendet werden, wenn man eines der konfligierenden Ziele aufgibt. Obwohl Klinger bereits 1977 eine Stufentheorie der Ablösung von Anreizen vorstelite, steht die systematische Analyse der Ablösung von gesetzten Zielen noch aus. Das bloße Grübeln über das, was der Zielerreichung im Wege steht, ist nicht genug (Martin \& Tesser, 1996; Oettingen, 1996, 1997a). Um ein aktives Ablösen einzuleiten müssen bestehende niedrige Erfolgserwartungen aktiviert werden, und dies geschieht immer dann, wenn die relevante erwünschte Zukunft mit der widersprechenden Realität mental kontrastiert wird.

\section{Literatur}

Ach, N. (1935). Analyse des Willens. In E. Abderhalden (Ed.), Handbuch der biologischen Arbeitsmethoden (Vol. 6). Berlin: Urban \& Schwarzenberg.

Ajzen, l. (1985). From intentions to actions: A theory of planned behavior. In J. Kuhl \& J. Beckmann (Eds.), Action control: Text Pl From cognition to behavior (pp. 11-39). Heidelberg: Springer- Verlag.

Ames, C. \& Archer, J. (1988). Achievement goals in the classroom: Students' learning strategies and motivation processes. Journal of Educational Psychology, 80, 260-267.

Atkinson, J. W. (1957). Motivational determinants of risk-taking behavior. Psychological Review, 64, 359-372.

Austin, J. T. \& Vancouver, J. B. (1996). Goal constructs in psychology: Structure, process, and content. Psychological Bulletin, 120, 338-375.

Bandura, A. (1997). Self-efficacy: The exercise of control. New York: Freeman.

Bandura, A. \& Schunk, D. H. (1981). Cultivating competence, self-efficacy and intrinsic interest through proximal selfmotivation. Journal of Personality and Social Psychology, 41, 586-598.

Bargh, J. A. (1990). Auto-motives: Pre-conscious determinants of social interaction. In E. T. Higgins \& R. M. Sorrentino (Eds.), Handbook of motivation and cognition (Vol. 2, pp. 93-130). New York: Guilford.

Bargh, J. A., Gollwitzer, P. M., Lee Chai, A. \& Barndollar, K. (1999). Bypassing the will: Nonconscious self-regulation through automatic goal pursuit. Manuscript submitted for publication.

Bindra, D. (1959). Motivation. New York: Ronald Press.

Brehm, J. W. \& Self, E. A. (1989). The intensity of motivation. Annual Review of Psychology, 45, 560-570.

Brooks, R. A. (1991). New approaches to robotics. Science, 253, 1227-1232.

Text P]

Text $\mathrm{Pl}$

Text PI]

Text P!

Text Pl

Text $P 1$

Brunstein, J. C., Schultheiss, O. C. \& Maier, G. W. (1999). The pursuit of personal goals: A motivational approach to wellbeing and life adjustment. In J. Brandtstädter \& R. M. Lerner (Eds.), Action and self-development: Theory and research through the life span (pp. 169-198). Thousand Oaks, CA: Sage.

Cantor, N. \& Fleeson, W. (1991). Life tasks and self-regulatory processes. In M. Maehr \& P. Pintrich (Eds.), Advances in motivation and achievement (Vol. 7, pp. 327-369). Greenwich, CT: JAl Press.

Cantor, N. \& Fleeson, W. (1994). Social intelligence and intelligent goal pursuit: A cognitive slice of motivation. In W. Spaulding (Ed.), Nebraska Symposium on Motivation (Vol. 41, pp. 125-180). Lincoln: University of Nebraska Press. Carver, C. S. \& Scheier, M. F. (1998). On the self-regulation of behavior. New York: Cambridge University Press.

Carver, C. S. \& Scheier, M. F. (1999). Themes and issues in the self-regulation of behavior. In R. S. Wyer (Ed.), Advances in social cognition. Mahwah, $\mathrm{NJ}$ : Eribaum.

Chaiken, S. (1987). The heuristic model of persuasion. In M. P. Zanna, J. M. Olson \& C. P. Herman (Eds.), Social influence: The Ontario symposium (Vol. 5, pp. 3-39). Hillsdale, NJ: Erlbaum.

Cochran, W. \& Tesser, A. (1996). The "what the hell" effect: Some effects of goal proximity and goal framing on performance. In L. L. Martin \& A. Tesser (Eds.), Striving and feeling (pp. 99-120). Mahwah, NJ: Erlbaum.

Bindra, D. (1959). Motivation. New York: Ronald Press.

Dweck, C. S. (1996). Implicit theories as organizers of goals and behavior. In P. M. Goliwitzer \& J. A. Bargh (Eds.), The psychology of action: Linking cognition and motivation to behavior (pp. 69-90). New York: Guilford.

Elliot, A. J. \& Church, M. A. (1997). A hierarchical model of approach and avoidance achievement motivation. Journal of Personality and Social Psychology, 72, 218-232.

Emmons, R. A. (1989). The personal striving approach to personality. In L. A. Pervin (Ed.), Goal concepts in personality and social psychology (pp. 87-126). Hilldale, NJ: Erlbaum.

Emmons, R. A. (1996). Striving and feeling: Personal goals and subjective weil-being. In P. M. Gollwitzer \& J. A. Bargh (Eds.), The psychology of action: Linking cognition and motivation to behavior (pp. 313-337). New York: Guilford Press. Festinger, L. (1942). A theoretical interpretation of shifts in level of aspiration. Psychological Review, 49, 235-250.

Frese, M. \& Sabini, J. (1985). Goal-directed behavior: The concept of action in psychology. Hillsdale, NJ: Erlbaum. Geen, R. G. (1995). Human motivation. Pacific Grove, CA: Brooks/Cole Publishing Company.

Gollwitzer, P. M. (1987). The implementation of identity intentions. In F. Halisch \& J. Kuhl (Eds.), Motivation, intention, and action. Berlin: Springer.

Gollwitzer, P. M. (1990). Action phases and mind-sets. In E. T. Higgins \& R. M. Sorrentino (Eds.), Handbook of 
motivation and cognition (Vol. 2, pp. 53-92). New York: Guilford.

Gollwitzer, P. M. (1993). Goal achievement: The role of intentions. In W. Stroebe \& M. Hewstone (Eds.), European Review of Social Psychology (Vol. 4, pp. 141-185). Chichester, England: John Wiley.

Gollwitzer, P. M. (1999). Implementation intentions: Strong effects of simple plans. American Psychologist, 54, 493-503.

Gollwitzer, P. M. \& Bargh, J. A., Eds. (1996). The psychology of action: Linking cognition and motivation to behavior. New York: Guilford.

Gollwitzer, P. M. \& Bayer, U. (1999). Deliberative versus implemental mindsets in the control of action. In S. Chaiken \& Y. Trope (Eds.), Dual process theories in social psychology (pp. 403-422). New York: Guilford.

Gollwitzer, P. M., Bayer, U., Scherer, M. \& Seifert, A. E. (1999). A motivational- volitional perspective on identity development. In J. Brandtstädter \& R. M. Lerner (Eds.), Action and self-development: Theory and research through the life span (pp. 283-314). Thousand Oaks, CA: Sage.

Goliwitzer, P. M., Heckhausen, H. \& Ratajczak, H. (1990). From weighing to willing: Approaching a change decision through pre- or postdecisional mentation. Organizational Behavior and Human Decision Processes, 45, 41-65.

Gollwitzer, P. M. \& Kirchhof, O. (1998). The willful pursuit of identity. In J. Heckhausen \& C. S. Dweck (Eds.), Motivation and self-regulation across the life-span (pp. 389-423). New York: Cambridge University Press.

Gollwitzer, P. M. \& Moskowitz, G. B. (1996). Goal effects on action and cognition. In E. T. Higgins \& A. W. Kruglanski (Eds.), Social psychology: A handbook of basic principles (pp. 361-399). New York: Guilford Press.

Gollwitzer, P. M. \& Schaal, B. (1998). Metacognition in action: The importance of implementation intentions. Personality and Social Psychology Review, 2, 124-136.

Gollwitzer, P. M. \& Rohloff, U. (1999). The speed of goal pursuit. In R. S. Wyer (Ed.), Advances in Social Cognition (Vol. 12, pp. 147-159). Hillsdale, NJ: Erlbaum.

Heckhausen, H. (1977). Achievement motivation and its constructs: A cognitive model. Motivation and Emotion, 1, 283329.

Heckhausen, H. (1991). Motivation and action. Heidelberg: Springer-Verlag.

Heckhausen, H. \& Golwwitzer, P. M. (1987). Thought contents and cognitive functioning in motivational versus volitional states of mind. Motivation and Emotion, 11, 101-120.

Heckhausen, H. \& Kuhl, J. (1985). From wishes to action: The dead ends and short cuts on the long way to action. In M. Frese \& J. Sabini (Eds.), Goal-directed behavior: The concept of action in psychology (pp. 134-159). Hilisdale, NJ:

Erlbaum.

Higgins, E. T. (1997). Beyond pleasure and pain. American Psychologist, 52, 1280-1300.

James, W. (1890). (1950). Principles of psychology (2 vols.). New York: Dover.

Karniol, R. \& Ross, M. (1996). The motivational impact of temporal focus: Thinking about the future and the past. Annual Review of Psychology, 47, 593-620.

Karoly, P. (1993). Mechanisms of self-regulation: A systems view. Annual Review of Psychology, 44, 23-52.

Kasser, T. \& Ryan, R. M. (1993). A dark side of the American dream: Correlates of financial success as a central life aspiration. Journal of Personality and Social Psychology, 65, 410-422.

Kelso, J. A. S. (1995). Dynamic patterns: The self-organization of brain and behavior. Cambridge, MA: MIT Press.

Klinger, E. (1977). Meaning and void. Minneapolis: University of Minnesota Press.

Kuhl, J. (1984). Volitional aspects of achievement motivation and learned helplessness: Toward a comprehensive theory of action control. In B. A. Maher \& W. A. Maher (Eds.), Progress in experimental personality research (pp. 99-171). New York: Academic Press.

Kuhl, J. (2000). A functional-design approach to motivation and self-regulation: The dynamics of personality systems interactions. In M. Boekaerts, P. R. Pintrich \& M. Zeidner (Eds.), Handbook of self-regulation. New York: Academic Press.

Kuhl, J. \& Beckmann, J. (1994). Volition and personality. Göttingen: Hogrefe.

Lewin, K. (1926). Vorsatz, Wille und Bedürfnis. Psychologische Forschung, 7, 330-385.

Text Pl

Text Pl

Text Pl

Text $\mathrm{Pl}$

Text $\mathrm{Pl}$

Text PI

Text $\mathrm{Pl}$

Text Pl

Text PI

Text PI

Text $\mathrm{Pl}$

Text PI

Text PI

Text PI

Text Pl

Text P]

Texi $\mathrm{Pl}$

Text $\mathrm{Pl}$

Text Pl

Text $\mathrm{Pl}$

Text Pl

Text PI

Little, B. R. (1983). Personal projects: A rationale and methods for investigation. Environment and Behavior, 15, 273-309. Locke, E. A. \& Latham, G. P. (1990). A theory of goal setting and task performance. Englewood Cliffs, NJ: Prentice Hall. Maes, P. (1994). Modeling adaptive autonomous agents. Artificial Life, 1, 135-162.

Markus, H. \& Nurius, P. (1986). Possible selves. American Psychologist, 41, 954-969.

Martin, L. L. \& Tesser, A. (1996). Some ruminative thoughts. In R. S. Wyer (Ed.), Advances in Social Cognition (Vol. 9 , pp. 1-47). Mahwah, NJ: Erlbaum.

McClelland, D. C. (1985). Human motivation. Glenview, Ill.: Scott, Foresman, \& Co..

Text Pl

McClelland, D. C., Koestner, R. \& Weinberger, J. (1989). How do self-attributed and implicit motives differ?.

Psychological Review, 96, 690-702.

McDougall, W. (1908). (1931). Social Psychology. London: Methuen.

McGuire, W. J. (1969). The nature of attitudes and attitude change. In G. Lindzey \& E. Aronson (Eds.), Handbook of social psychology (2nd ed., Vol. 3, pp. 136-314). Reading, MA: Addison-Wesley. Mischel, W., Cantor, N. \& Feldman, S. (1996). Principles of self-regulation: The nature of willpower and self-control. In E. 
T. Higgins \& A. W. Kruglanski (Eds.), Social psychology: A handbook of basic principles (pp. 329-360). New York: Guilford Press.

Mischel, W. \& Liebert, R. M. (1966). Effects of discrepancies between observed and imposed reward criteria on their acquisition and transmission. Journal of Personality and Social Psychology, 3, 45-53.

Nicholls, J. G. (1979). Quality and equality in intellectual development: The role of motivation in education. American Psychologist, 34, 1071-1084.

Oettingen, G. (1996). Positive fantasy and motivation. In P. M. Gollwitzer \& J. A. Bargh (Eds.), The psychology of action: Linking cognition and motivation to behavior (pp. 236-259). New York: Guilford.

Oettingen, G. (1997 a). Psychologie des Zukunftsdenkens: Erwartungen und Phantasien. Göttingen: Hogrefe.

Oettingen, G. (1997 b). Culture and future thought. Culture and Psychology, 3, 353-381.

Oettingen, G. (1999). Free fantasies about the future and the emergence of developmental goals. In J. Brandtstädter \& R. M. Lerner (Eds.), Action and self-development: Theory and research through the life span (pp. 315-342). Thousand Oaks, CA: Sage.

Oettingen, G. (2000). Expectancy effects on behavior depend on self-regulatory thought. Social Cognition, 18, $101-129$. Pervin, L. A., Ed. (1989). Goal concepts in personality and social psychology. Hillsdale, NJ: Erlbaum.

Petty, R. E. \& Cacioppo, J. T. (1986). Communication and persuasion: Central and peripheral routes to attitude change. New York: Springer Verlag.

Read, S. J., Vanman, E. J. \& Miller, L. C. (1997). Connectionism, parallel constraint satisfaction processes, and Gestalt principles: (Re)introducing cognitive dynamics to social psychology. Review of Personality and Social Psychology, 1, 2653.

Ryan, R. M.; Sheldon, K. M., Kasser, T. \& Deci, E. L. (1996). All goals are not created equal: an organismic perspective on the nature of goals and their regulation. In P. M. Goliwitzer \& J. A. Bargh (Eds.), The psychology of action: Linking cognition and motivation to behavior (pp. 7-26). New York: Guilford.

Scheier, M. F. \& Carver, C. S. (1985). Optimism, coping, and health: Assessment and implications of generalized outcome expectancies. Health Psychology, 4, 219-247.

Skinner, B. F. (1953). Science and human behavior. New York: Macmillan.

Steele, C. M. (1988). The psychology of self-affirmation: Sustaining the integrity of the self. In L. Berkowitz (Ed.), Advances in Experimental Social Psychology (Vol. 21, pp. 261-302). New York: Academic Press.

Taylor, S. E., Pham, L. B., Rivkin, I. D. \& Armor, D. A. (1998). Harnessing the imagination. American Psychologist, 53, 429-439.

Tesser, A., Martin, L. L. \& Cornell, D. P. (1996). On the substitutability of self-protective mechanisms. In P. M. Gollwitzer \& J. A. Bargh (Eds.), The psychology of action. Linking cognition and motivation to behavior (pp. 48-67). New York: Guilford.

Tolman, E. C. (1925). Purpose and cognition: The determinants of animal learning. Psychological Review, 32, $285-297$. Vallacher, R. R. \& Wegner, D. M. (1987). What do people think they're doing? Action identification and human behavior. Psychological Review, 94, 3-15.

Valiacher, R. R. \& Wegner, D. M. (1989). Levels of personal agency: Individual variation in action identification. Journal of Personality and Social Psychology, 57, 660-671.

Wicklund, R. A. \& Gollwitzer, P. M. (1982). Symbolic self-completion. Hillsdale, NJ: Erlbaum.

Wilpert, B. (1994). Participation research in organizational psychology. In G. d'Ydewalle, P. Eelen \& P. Berteison (Eds.), International perspectives on psychological science (Vol. 2, pp. 293-310). Howe, England: Erlbaum.

Wright, R. A. (1996). Brehm's theory of motivation as a model of effort and cardiovascular response. In P. M. Goilwitzer \& J. A. Bargh (Eds.), The psychology of action: Linking cognition and motivation to behavior (pp. 424-453). New York: Guilford.

Wright, R. A., Murray, J. B., Storey, P. L. \& Williams, B. J. (1997). Ability analysis of gender relevance and sex differences in cardiovascular response to behavioral challenge. Journal of Personality and Social Psychology, 73, 405417.

Wright, R. A., Tunstall, A. M., Williams, B. J., Goodwin, J. S. \& Harmon-Jones, E. (1995). Social evaluation and cardiovascular response: An active coping approach. Journal of Personality and Social Psychology, 69, 530-543.

\section{Anschrift}

Gabriele Oettingen, Prof., Dr., Universität Hamburg, Psychologisches Institut II, Von-Melle-Park 5, D-20146, Hamburg, gaboettingen@aol.com.

Peter M. Gollwitzer, Prof., Dr., Universität Konstanz, Fachgruppe Psychologie, Postfach D 39, D-78457, Konstanz, peter.gollwitzer@uni-konstanz.de. 